\title{
Determination of Risk Factors for Pain After Transarterial Chemoembolization with Drug-Eluting Beads for Hepatocellular Carcinoma
}

This article was published in the following Dove Press journal: Journal of Pain Research

\section{Tian-Cheng Wang Zi-Shu Zhang \\ Yu-Dong Xiao (D)}

Department of Radiology, The Second Xiangya Hospital of Central South University, Changsha, Hunan, People's Republic of China
Correspondence: Yu-Dong Xiao Email xiaoyudong222@csu.edu.cn
Purpose: To identify risk factors for pain after transarterial chemoembolization with drugeluting beads (DEB-TACE) for hepatocellular carcinoma (HCC).

Patients and Methods: In this retrospective study, a total of 118 consecutive patients who underwent DEB-TACE between June 2016 and May 2019 with post-TACE pain were included. The patients were divided into three groups based on the severity of post-TACE pain according to the distribution of pain Visual Analogue Scale/Score (VAS). Potential risk factors for post-TACE pain were primarily analyzed using the chi-square test, one-way analysis of variance, or KruskalWallis test (if appropriate). For multivariate analysis, an ordinal logistic regression model was utilized. Variables with $\mathrm{P}<0.10$ in the univariate analysis were included in a multivariate model to identify independent risk factors for post-TACE pain. A multivariate analysis was also performed by means of a decision tree using the Classification and Regression Tree (CART) algorithm.

Results: The univariate analysis showed that elderly patients or patients with portal venous tumor thrombus (PVTT) were more likely to have severe post-TACE pain than young patients or those without PVTT $(\mathrm{P}=0.028$ and $<0.001$, respectively). However, in the ordinal logistic regression, nonsuperselective chemoembolization and presence of PVTT were independent risk factors of severe post-TACE pain $(\mathrm{P}=0.046$ and $<0.001$, respectively). In addition, the CART showed that nonsuperselective chemoembolization and PVTT could increase the probability of severe post-TACE pain.

Conclusion: Nonsuperselective chemoembolization and PVTT are independent risk factors for pain after DEB-TACE. Therefore, these factors should be taken into full consideration for the relief of pain.

Keywords: microspheres, chemoembolization, therapeutic, risk factors, carcinoma, hepatocellular, pain

\section{Introduction}

Hepatocellular carcinoma (HCC) is the sixth most common malignancy and represents the third leading cause of cancer death worldwide. ${ }^{1-3}$ For patients with HCC, surgical resection, ablative therapy, and liver transplantation are the potentially curative treatments. $^{4-6}$ Transarterial chemoembolization (TACE) is the standard and effective therapy for intermediate or advanced-stage HCC. $^{7}$ Although TACE is related to a significant increase in overall survival compared with the best supportive care, ${ }^{8,9}$ it is related to a high rate of side effects, which is also referred to as postembolization syndromes including fever, abdominal pain, and nausea/vomiting. ${ }^{10}$ Of all postembolization syndromes, pain appears most frequently and is associated with an extended 
hospital stay. ${ }^{11,12}$ The post-TACE pain is well documented in patients treated with conventional TACE (cTACE), ${ }^{13-15}$ however, it is not well studied in patients treated with drugeluting beads TACE (DEB-TACE). Lipiodol and DEB are completely different materials for embolization because lipiodol can pass through the hepatic sinusoid into the portal vein while DEB cannot. Additionally, it has been suggested that DEB-TACE can offer a better safety profile with a lower incidence of postembolization syndrome and drug-related systemic toxicity than cTACE. ${ }^{16-18}$ Thus, we believe that the risk factors for pain after cTACE and DEB-TACE are quite different.

Therefore, the purpose of the present study was to investigate the risk factors for pain in HCC patients after DEB-TACE.

\section{Materials and Methods}

\section{Patients}

This retrospective study was approved by the institutional review board of the Second Xiangya Hospital of Central South University in accordance with Declaration of Helsinki. The requirement for written informed consent was waived by the institutional review board due to the retrospective nature of the study.

A total of 289 consecutive patients with unresectable hepatic malignancy who underwent DEB-TACE and had post-TACE pain were included in this retrospective study between March 2016 and May 2019. The inclusion criteria of DEB-TACE were as follows: (1) Eastern Cooperative Oncology Group (ECOG) performance status 0-1; (2) international normalized ratio $<1.5$ and platelet count $>50,000$ / $\mathrm{mm}^{3}$; (3) compensated liver function (Child-Pugh class A or B); (4) no refractory ascites or renal failure. The exclusion criteria were as follows: (1) diagnosed hepatic malignancy other than HCC $(n=84)$; (2) portal venous tumor thrombus (PVTT) in the main portal vein or bilateral portal branches $(n=49)$; (3) uncontrolled or significant cardiovascular disease $(\mathrm{n}=15)$; (4) administrating non-steroidal anti-inflammatory drugs or steroids periodically $(\mathrm{n}=12)$; (5) uncontrollable diabetes mellitus with $\mathrm{HbA} 1 \mathrm{c} \geq 8.0 \mathrm{~g} / \mathrm{dL}(\mathrm{n}=11)$. Finally, a total of 118 patients with unresectable HCC were included (105 males and 13 females, with a mean age of $55.2 \pm 10.3$ years). The diagram of the study population is shown in Figure 1.

\section{Interventions}

The procedure was performed by three interventional radiologists with 17 years, 21 years and 13 years of experience

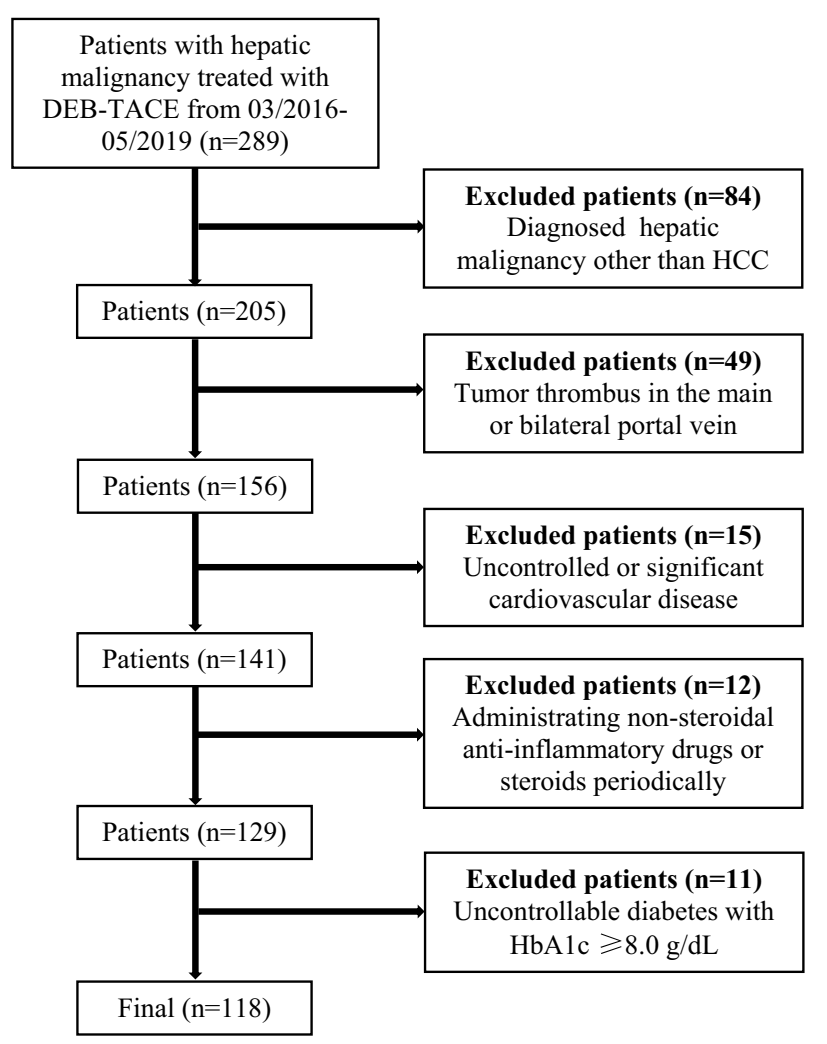

Figure I Diagram of the study population.

in liver interventions, respectively. Celiac trunk arteriography and superior mesenteric arteriography, as well as indirect portography, were performed to visualize the variations in hepatic arterial anatomy and evaluate the patency of the portal vein. A 2.2 French coaxial microcatheter (Carnelian, Tokai Medical Products, Aichi, Japan) was used to select the branch of the hepatic artery that supplied the target tumor, and cone-beam computed tomography was used to reconfirm the location of HCC lesions if necessary. Before embolization, midazolam and fentanyl were routinely used tailored individually to body habitus and drug tolerance for moderate sedation and $10 \mathrm{mg}$ dexamethasone was used for prophylactic reducing TACEinduced nausea/vomiting. Patients were intra-arterially injected with 1-2 g DEB (CalliSpheres Beads, Jiangsu Hengrui Medicine Co. Ltd). Size of the DEB varied from $100 \mu \mathrm{m}$ to $700 \mu \mathrm{m}$, and the amount of epirubicin used ranged from $40 \mathrm{mg}$ to $120 \mathrm{mg}$. A substantial reduction in the arterial flow to the tumor was the technical endpoint, whereas complete occlusion was avoided. Repeated hepatic arteriography was performed to assess devascularization after embolization. Chemoembolization was performed as superselectively as possible. The definition of 
superselective TACE was chemoembolization at the subsegmental hepatic artery. ${ }^{19}$

\section{Data Collection}

In our institution, the degree of pain and the analgesics used after TACE were recorded in the electronic medical records. Because pain usually occurs and reaches a peak within 12-24 hrs after embolization, the post-TACE pain is recorded within $24 \mathrm{~h}$ after the procedure. ${ }^{20}$ Patients with post-TACE pain were divided into three groups based on the distribution of pain Visual Analogue Scale/Score (VAS): mild pain (5-44 mm, Group A, $\mathrm{n}=63$ ), moderate pain (45-74 mm, Group $B, n=26)$, and severe pain (75-100 mm, Group C, n=29). ${ }^{21}$

The demographic, laboratory, and radiological data of patients before TACE were collected to assess the potential risk factors for post-TACE pain. The demographic data included age, sex, Child-Pugh class, ECOG status, underlying liver disease, dose of epirubicin administered, size of DEB used, and history of hepatectomy. The laboratory data included serum alpha-fetoprotein, albumin, and total bilirubin levels. The radiological data included the presence or absence of PVTT, hepatic arterio-portal shunt (HAPS), size of the largest tumor, whether the lesion was adjacent to the liver capsule, ${ }^{22}$ extent of tumor involved (right lobe, left lobe, or bilobar), number of lesions, superselective or nonsuperselective chemoembolization during the procedure. The radiological data were independently reviewed by two radiologists with either 21- or 18-year experience of abdominal imaging. They were blinded to the demographic and laboratory data, and not involved in the treatment. The final results of radiological data were made by the discussion of two radiologists.

\section{Statistical Analysis}

The data were shown as the mean with standard deviation (SD), median with interquartile range (IQR), or frequency. In order to evaluate the inter-reader agreement of radiological data between two radiologists, either intraclass correlation coefficient (ICC) (for numerical data) or Kappa test (for categorical data) was performed. Agreement was classified as poor (ICC or Kappa value, 0-0.40), fair to good (ICC or Kappa value, 0.40-0.75), and excellent (ICC or Kappa value, $>0.75$ ). In the univariate analyses, Pearson's chi-squared test or Fisher's exact test was used to compare categorical variables, while the one-way analysis of variance was used to compare numerical variables.
In the multivariate analysis, an ordinal logistic regression model was used. Variables with a P-value less than 0.10 in the univariate analysis were included in the multivariate model. A multivariate analysis was also performed to assess the risk factors that affected the probability of severe post-TACE pain by means of a decision tree using the Classification and Regression Tree (CART) algorithm. All statistical analyses were performed by a statistics package (SPSS version 20, International Business Machines Corporation), and $\mathrm{P}<0.05$ was considered statistically significant.

\section{Results}

\section{Demographic and Laboratorial Characteristics}

All 118 patients had mild to severe post-TACE pain. According to the distribution of pain VAS, there were 63 patients with mild pain in group A, 26 patients with moderate pain in group B, and 29 patients with severe pain in group $\mathrm{C}$. The diagnosis of HCC was based on pathology (biopsy, $n=16$ ) or on the American Association for the Study of Liver Practice Guidelines $(n=102)$. Of the 118 patients, 105 patients had a history of cirrhosis, and 13 patients were noncirrhotic. The etiologies of liver cirrhosis were hepatitis B infection $(n=104,88.1 \%)$ and hepatitis $\mathrm{C}$ infection $(\mathrm{n}=1,0.8 \%)$. There were 97 patients $(82.2 \%)$ in Child-Pugh class A and 21 patients (17.8\%) in ChildPugh class B. The median serum total bilirubin was 17.6 $\mu \mathrm{mol} / \mathrm{L}$ (IQR $11.5-24.4 \mu \mathrm{mol} / \mathrm{L}$ ), and the median albumin was $33.3 \mathrm{~g} / \mathrm{L}$ (IQR 30.4-36.0 g/L). The detailed demographic and laboratory data of all patients are listed in Table 1.

\section{Radiological Characteristics}

The inter-reader agreements of radiological data between two radiologists were all excellent with Kappa values of 0.976 (presence or absence of unilateral PVTT), 0.947 (presence or absence of HAPS), 0.813 (whether the lesion adjacent to the liver capsule), 0.961 (extent of tumor involved), and ICC value of 0.838 (size of the largest tumor)

All DEB-TACE procedures achieved technical success according to the Society of Interventional Radiology (SIR) guidelines. ${ }^{23}$ The median largest tumor diameter was $6.25 \mathrm{~cm}$ (IQR 4-10 cm). Six out of $118(6 / 118,5.1 \%)$ patients had tumors involved in the left lobe of the liver, $97(97 / 118$, $82.2 \%$ ) had tumors involved the right lobe of the liver, and 
Table I The Demographic and Laboratorial Characteristics of All Patients

\begin{tabular}{|c|c|}
\hline Characteristics & \\
\hline Age (years) & $55.24 \pm 10.27$ \\
\hline Gender (male/female) & $105 / 13(89.0 \% / 11.0 \%)$ \\
\hline Child-Pugh class (A/B) & $97 / 21(82.2 \% / 17.8 \%)$ \\
\hline ECOG status $(0 / 1)$ & $97 / 21(82.2 \% / 17.8 \%)$ \\
\hline Underlying liver disease (HBV/HCV/None) & $\begin{array}{l}104 / 1 / 13(88.1 \% / 0.8 \% / \\
11.1 \%)\end{array}$ \\
\hline Dose of epirubicin administrated (mg) & 60 (IQR 60-80) \\
\hline $\begin{array}{l}\text { Size of DEB ( } 100-300 \mathrm{um} / 300-500 \\
\text { um/500-700 um) }\end{array}$ & $\begin{array}{l}38 / 74 / 6(32.2 \% / 62.7 \% / \\
5.1 \%)\end{array}$ \\
\hline $\begin{array}{l}\text { History of hepatectomy (presence/ } \\
\text { absence) }\end{array}$ & $10 / 108$ (8.5\%/9I.5\%) \\
\hline Albumin $(g / L)$ & 33.3 (IQR 30.4-36.0) \\
\hline Alpha-fetoprotein (>400 ug/L/ $\leq 400 \mathrm{ug} / \mathrm{L}$ ) & $59 / 59(50.0 \% / 50.0 \%)$ \\
\hline Total serum bilirubin (umol/L) & I7.6 (IQR II.5-24.4) \\
\hline
\end{tabular}

Abbreviations: ECOG, eastern cooperative oncology group; HBV, hepatitis $B$ virus; $H C V$, hepatitis $C$ virus; IQR, interquartile range; $D E B$, drug-eluting beads.

$15(15 / 118,12.7 \%)$ had tumors with bilobar involvement. Thirty-eight patients $(38 / 118,32.2 \%)$ had PVTT, and 80 patients $(80 / 118,67.8 \%)$ did not have PVTT. Twenty-eight patients $(28 / 118,23.7 \%)$ were treated with superselective TACE, 90 patients $(90 / 118,76.3 \%)$ were treated with nonsuperselective TACE. Sixty-four patients were presence of HAPS, and 54 patients were absence of HAPS. The detailed radiological characteristics are summarized in Table 2.

\section{Potential Risk Factors for Post-TACE Pain}

The results of the univariate analysis for post-TACE pain are illustrated in Table 3. Post-TACE pain was associated with PVTT $(\mathrm{P}<0.001)$ and age $(\mathrm{P}=0.028)$. There was no statistical relationship between post-TACE pain and sex, Child-Pugh class, ECOG status, underlying liver disease, history of hepatectomy, size of the largest tumor, whether the lesion was adjacent to the liver capsule, serum alphafetoprotein level, albumin level, total serum bilirubin level, extent of tumor involved, number of lesions, dose of epirubicin administered, size of DEB used, HAPS, and superselective or nonsuperselective chemoembolization.

Because the results of the univariate analysis showed that the differences in age, PVTT, and superselective or nonsuperselective chemoembolization among the three groups had
Table 2 The Radiological Characteristics of All Patients

\begin{tabular}{|l|l|}
\hline Characteristics & \\
\hline $\begin{array}{l}\text { Size of the largest tumor (cm) } \\
\text { Tumor involvement scope (left/right/ } \\
\text { bilobar) }\end{array}$ & $\begin{array}{l}6.25(\mathrm{IQR} 4-10) \\
6 / 97 / 15(5.1 \% / 82.2 \% / \\
12.7 \%)\end{array}$ \\
\hline Number of lesions (I/2-3/>3) & $\begin{array}{l}10 / 35 / 73(8.4 \% / 29.7 \% / \\
61.9 \%)\end{array}$ \\
\hline PVTT (presence/absence) & $38 / 80(32.2 \% / 67.8 \%)$ \\
\hline $\begin{array}{l}\text { Lesion adjacent to liver capsule (presence/ } \\
\text { absence) }\end{array}$ & $48 / 70(40.7 \% / 59.3 \%)$ \\
\hline $\begin{array}{l}\text { Superselective chemoembolization } \\
\text { (presence/absence) }\end{array}$ & $28 / 90(23.7 \% / 76.3 \%)$ \\
\hline HAPS (presence/absence) & $64 / 54(54.2 \% / 45.8 \%)$ \\
\hline
\end{tabular}

Abbreviations: IQR, interquartile range; PVTT, portal venous tumor thrombus; HAPS, hepatic arterio-portal shunt.

$P$ values less than 0.10 , these three factors were included in the multivariate analysis. In multivariate analysis, PVTT $(\mathrm{P}<0.001$; odds ratio $=4.61,95 \% \mathrm{CI}, 2.094-10.196)$ and nonsuperselective chemoembolization $\quad(\mathrm{P}=0.046 ; \quad$ odds ratio $=2.838,95 \%$ CI, 1.018-7.909) were the independent risk factors associated with post-TACE pain.

The factors associated with post-TACE pain in the multivariate analysis were put into the CART algorithm to assess the probability of post-TACE pain (Figure 2). Because patients in group $\mathrm{B}$ or group $\mathrm{C}$ often needed analgesic therapy, we combined group $\mathrm{B}$ and group $\mathrm{C}$ into a single group for the CART analysis. As shown in Figure 2, patients with PVTT presented a $76.3 \%$ probability of having moderate to severe post-TACE pain. However, patients without PVTT presented with a low probability of moderate to severe post-TACE pain (32.5\%). In patients with PVTT, nonsuperselective chemoembolization increased the probability of moderate to severe post-TACE pain from $60.0 \%$ to $78.8 \%$. Meanwhile, in patients without PVTT, nonsuperselective chemoembolization only increased the probability of moderate to severe post-TACE pain from $13.6 \%$ to $39.7 \%$. The model was presented with good adjustment with an estimated risk of 0.30 , indicating that the CART decision tree was able to correctly classify $70 \%$ of the data variability.

\section{Discussion}

Pain is a common complication after TACE and is considered to be related to the distention of the liver capsule, tumor necrosis, acute liver parenchymal ischemia, and inadvertent embolization of the cystic artery. ${ }^{24}$ Although post-TACE 
Table 3 Assessment of Potential Risk Factors of Post-TACE Pain

\begin{tabular}{|c|c|c|c|c|c|}
\hline \multirow[t]{2}{*}{ Characteristics } & \multirow[t]{2}{*}{ Group A $(n=63)$} & \multirow[t]{2}{*}{ Group B $(n=26)$} & \multirow[t]{2}{*}{ Group C $(n=29)$} & \multicolumn{2}{|l|}{$P$ value } \\
\hline & & & & Univariate & Multivariate \\
\hline Age (years) & $57.14 \pm 10.13$ & $55.35 \pm 9.35$ & $51.03 \pm 10.45$ & 0.028 & 0.19 \\
\hline Gender (male/female) & $55 / 8(87.3 \% / 12.7 \%)$ & $24 / 2(92.3 \% / 7.7 \%)$ & $26 / 3(89.7 \% / 10.3 \%)$ & 0.783 & - \\
\hline Child-Pugh class (A/B) & $52 / 11(82.5 \% / 17.5 \%)$ & $19 / 7(73.1 \% / 26.9 \%)$ & $26 / 3(89.7 \% / 10.3 \%)$ & 0.274 & - \\
\hline ECOG status $0 / 1$ & $52 / 11(82.5 \% / 17.5 \%)$ & $20 / 6(77.0 \% / 23.0 \%)$ & $25 / 4(86.2 \% / 13.8 \%)$ & 0.664 & - \\
\hline Underlying liver disease $\mathrm{HBV} / \mathrm{HCV} /$ none & $\begin{array}{l}52 / 1 / 10(82.5 \% / 1.6 \% / \\
15.9 \%)\end{array}$ & $25 / 0 / 1(96.1 \% / 0 / 3.9 \%)$ & $27 / 0 / 2(93.1 \% / 0 / 6.9 \%)$ & 0.175 & - \\
\hline PVTT (presence/absence) & $9 / 54(14.3 \% / 85.7 \%)$ & $12 / 14(46.2 \% / 53.8 \%)$ & $17 / 12(58.6 \% / 4 \mid .4 \%)$ & $<0.001$ & $<0.001$ \\
\hline History of hepatectomy (presence/absence) & $4 / 59(6.3 \% / 93.7 \%)$ & $2 / 24(7.7 \% / 92.3 \%)$ & $4 / 25(13.8 \% / 86.2 \%)$ & 0.486 & - \\
\hline Largest tumor size $(\mathrm{cm})$ & 6.0 (IQR 3.8-9.7) & 7.5 (IQR 4.8-11.4) & 6.3 (IQR 4.3-10.5) & 0.324 & - \\
\hline $\begin{array}{l}\text { Tumor near liver capsule (presence/ } \\
\text { absence) }\end{array}$ & $24 / 39(38.1 \% / 61.9 \%)$ & $13 / 13(50.0 \% / 50.0 \%)$ & II/I8 (37.9\%/62.1\%) & 0.548 & - \\
\hline Alpha-fetoprotein $>400 \mathrm{ug} / \mathrm{L} / \leq 400 \mathrm{ug} / \mathrm{L}$ & $29 / 34(46.0 \% / 54.0 \%)$ & $17 / 9(65.4 \% / 34.6 \%)$ & 13/16 (44.8\%/55.2\%) & 0.205 & - \\
\hline Albumin $(g / L)$ & 33.5 (IQR 30.1-37.0) & 32.4 (IQR 29.7-35.4) & 34.1 (IQR 3I.5-36.0) & 0.536 & - \\
\hline Total bilirubin (umol/L) & I6.5 (IQR II.0-23.2) & I9.6 (IQR I2.5-27.4) & I8.5 (IQR I4.0-23.0) & 0.376 & - \\
\hline Tumor involved scope (left/right/bilobar) & $\begin{array}{l}1 / 52 / 10(1.6 \% / 82.5 \% / \\
15.9 \%)\end{array}$ & $\begin{array}{l}3 / 20 / 3(11.5 \% / 77.0 \% / \\
11.5 \%)\end{array}$ & $2 / 25 / 2(6.9 \% / 86.2 \% / 6.9 \%)$ & 0.26 & - \\
\hline Number of lesions $1 / 2-3 />3$ & $\begin{array}{l}6 / 23 / 34(9.5 \% / 36.5 \% / \\
54.0 \%)\end{array}$ & $2 / 6 / 18(7.7 \% / 23.1 \% / 69.2 \%)$ & $\begin{array}{l}2 / 6 / 21(6.9 \% / 20.7 \% / \\
72.4 \%)\end{array}$ & 0.443 & - \\
\hline Dose of epirubicin (mg) & 60 (IQR 60-80) & 60 (IQR 60-80) & 80 (IQR 60-80) & 0.712 & - \\
\hline $\begin{array}{l}\text { Size of DEB (um) } \\
100-300 / 300-500 / 500-700\end{array}$ & $\begin{array}{l}21 / 40 / 2(33.3 \% / 63.5 \% / \\
3.2 \%)\end{array}$ & $\begin{array}{l}10 / 14 / 2(38.5 \% / 53.8 \% / \\
7.7 \%)\end{array}$ & $\begin{array}{l}7 / 20 / 2(24.1 \% / 69.0 \% / \\
6.9 \%)\end{array}$ & 0.658 & - \\
\hline Superselective TACE (presence/absence) & $22 / 4 \mid(34.9 \% / 65.1 \%)$ & $3 / 23(11.5 \% / 88.5 \%)$ & $3 / 26(10.3 \% / 89.7 \%)$ & 0.052 & 0.046 \\
\hline HAPS (presence/absence) & $36 / 27$ (57.1\%/42.9\%) & |4/I2 (53.8\%/46.2\%) & |4/15 (48.3\%/5|.7\%) & 0.909 & - \\
\hline
\end{tabular}

Abbreviations: TACE, transarterial chemoembolization; ECOG, eastern cooperative oncology group; HBV, hepatitis $B$ virus; HCV, hepatitis C virus; PVTT, portal venous tumor thrombus; IQR, interquartile range; DEB, drug-eluting beads; HAPS, hepatic arterio-portal shunt; HCC, hepatocellular carcinoma; cTACE, conventional TACE; DEBTACE, drug-eluting beads TACE; VAS, Visual Analogue Scale/Score; SD, standard deviation; ICC, intraclass correlation coefficient; CART, Classification and Regression Tree, SIR, Society of Interventional Radiology.

pain is often mild and self-limited, it can significantly prolong the length of hospitalization and interfere with patient compliance. One of the multiple risk factors for post-TACE pain, as shown in the present study, is nonsuperselective chemoembolization. It has been reported that selective chemoembolization can significantly reduce post-TACE pain, ${ }^{13,25}$ and the present study showed a similar finding. A probable explanation of this phenomenon may be owing to the embolization-related hepatic ischemia. Compared to superselective chemoembolization, nonsuperselective procedure may result in more proximal embolization of the hepatic artery, which leads to ischemia of the liver parenchyma and causes severe pain. The other risk factor for post-TACE pain is the presence of PVTT. To the best of our knowledge, this is the first study to identify the presence of PVTT as a risk factor for post-TACE pain. It is a known factor that the liver has a dual blood supply from the portal vein ( $75 \%$ of blood flow) and hepatic artery ( $25 \%$ of blood flow) ${ }^{26}$ However, the blood flow in the liver may be changed in patients with PVTT. Usually, the hepatic artery will be dilated due to the presence of PVTT, and the blood flow will be increased to compensate for the lack of blood flow in the portal vein. ${ }^{27}$ Therefore, embolization of the hepatic artery in patients with PVTT may lead to more severe hepatic ischemia and cause more severe pain than in patients without PVTT. Third, in the univariate analysis, elderly patients were less likely to have 


\section{Probability of post-TACE pain in subgroups}

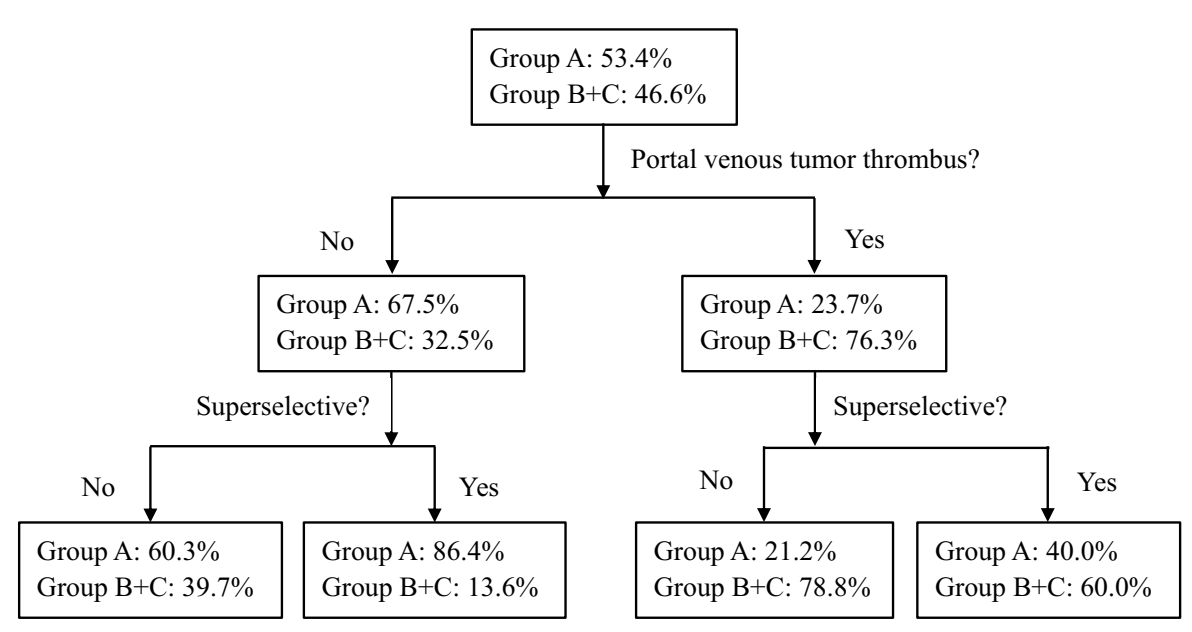

Figure 2 Probability of severe pain after transarterial chemoembolization (TACE) in subgroups. Patients with portal venous tumor thrombus (PVTT) presented a 76.3\% probability of having grade 2 and 3 post-TACE pain. However, patients without PVTT presented with a low probability of grade 2 and 3 post-TACE pain (32.5\%). In patients with PVTT, nonsuperselective chemoembolization increased the probability of grade 2 and 3 post-TACE pain from $60.0 \%$ to $78.8 \%$, while in patients without PVTT, nonsuperselective chemoembolization only increased the probability of grade 2 and 3 post-TACE pain from $13.6 \%$ to $39.7 \%$.

severe pain after TACE than younger patients, but this effect was not significant in the multivariate analysis. Although a previous study reported that elderly patients may have less pain after TACE than young patients, ${ }^{13}$ whether age can influence post-TACE pain is rather controversial. ${ }^{14,28}$ Thus, further prospective studies should be conducted to confirm this finding. Furthermore, the multivariate analysis in the present study using the CART algorithm showed similar risk factors and supported the findings from the ordinal logistic regression analysis. The CART algorithm showed that the presence of PVTT and nonsuperselective chemoembolization can increase the probability of moderate to severe post-TACE pain, which indicates that prophylactic analgesia should be taken into consideration in such patients.

Interestingly, underlying liver disease and whether the lesion adjacent to the liver capsule were not statistically correlated with post-TACE pain. Underlying liver disease was not a risk factor for post-TACE pain in the present study, which is not compatible with the results that Joseph's study showed. ${ }^{13}$ This discrepancy may be owing to the different study populations of the two studies. In the present study, the composition of patients was almost homogenous because the majority $(104 / 118,88.1 \%)$ had hepatitis B infections, while the population by Joseph et al was rather heterogeneous and included excessive alcohol consumption (14\%), hepatitis B infection (17\%), hepatitis C infection (27\%), nonalcoholic fatty liver disease $(10 \%)$, mixed causes $(22 \%)$, other causes $(4 \%)$, and no liver disease $(6 \%)$. We hypothesized that the embolization of lesions adjacent to the liver capsule may result in severe pain because the embolization may cause swelling of the liver and stretching of the capsule that contains the nerve fibers. However, we did not find any significant difference in post-TACE pain between patients with and without lesions located adjacent to the liver capsule. This factor was not related to post-TACE pain in Nilesh's study as well. ${ }^{15}$

There are several limitations in the present study. First, this is a retrospective study with a relatively small number of patients included and thus may be subject to selection and statistical bias. A prospective study with a relatively large study population should be performed to confirm this finding in the future. Second, pain is a subjective discomfort influenced by various factors. We used the distribution of pain VAS to divide patients into three groups instead of exact pain score. Third, we did not assess the relationship between the long-term outcomes of HCC patients and post-TACE pain. Although a previous study reported that postembolization syndrome is an early predictor of poor overall survival for patients with advanced and unresectable $\mathrm{HCC},{ }^{29}$ the relationship between post-TACE pain and overall survival has not been well studied. We believe this correlation needs to be further studied and characterized.

\section{Conclusion}

Nonsuperselective chemoembolization and PVTT are independent risk factors for pain after DEB-TACE. Further prospective investigations of the role of these factors in 
post-TACE pain should, therefore, be performed to improve the quality of pain management after DEB-TACE.

\section{Disclosure}

The authors report no conflicts of interest in this work.

\section{References}

1. Lazuardi F, Valencia J, Zheng S. Adjuvant transcatheter arterial chemoembolization after radical resection of hepatocellular carcinoma patients with tumor size less than $5 \mathrm{~cm}$ : a retrospective study. Scand J Gastroenterol. 2019;54(5):617-622. doi:10.1080/00365521. 2019.1607896

2. Chan KM, Cheng $\mathrm{CH}, \mathrm{Wu} \mathrm{TH}$, et al. Salvage living donor liver transplantation for posthepatectomy recurrence: a higher incidence of recurrence but promising strategy for long-term survival. Cancer Manag Res. 2019;11:7295-7305. doi:10.2147/CMAR.S215732

3. Young S, Craig P, Golzarian J. Current trends in the treatment of hepatocellular carcinoma with transarterial embolization: a cross-sectional survey of techniques. Eur Radiol. 2019;29 (6):3287-3295. doi:10.1007/s00330-018-5782-7

4. Kim W, Cho SK, Shin SW, Hyun D, Lee MW, Rhim H. Combination therapy of transarterial chemoembolization (TACE) and radiofrequency ablation (RFA) for small hepatocellular carcinoma: comparison with tace or RFA monotherapy. Abdom Radiol. 2019;44 (6):2283-2292. doi:10.1007/s00261-019-01952-1

5. Eilard MS, Andersson M, Naredi P, et al. A prospective clinical trial on sorafenib treatment of hepatocellular carcinoma before liver transplantation. BMC Cancer. 2019;19(1):568. doi:10.1186/s12885019-5760-8

6. Peng Z, Chen S, Xiao H, et al. Microvascular invasion as a predictor of response to treatment with sorafenib and transarterial chemoembolization for recurrent intermediate-stage hepatocellular carcinoma Radiology. 2019;292(1):237-247. doi:10.1148/radiol.2019181818

7. He M, Li Q, Shen J, et al. Predictive factors for the benefit of triple-drug transarterial chemoembolization for patients with unresectable hepatocellular carcinoma. Cancer Med-US. 2019;8 (9):4200-4213. doi:10.1002/cam4.2355

8. Lee DH, Lee JM, Kim PN, et al. Whole tumor ablation of locally recurred hepatocellular carcinoma including retained iodized oil after transarterial chemoembolization improves progression-free survival. Eur Radiol. 2019;29(9):5052-5062. doi:10.1007/s00330-018-5993-y

9. Piscaglia F, Ogasawara S. Patient selection for transarterial chemoembolization in hepatocellular carcinoma: importance of benefit/risk assessment. Liver Cancer. 2018;7(1):104-119. doi:10.1159/000485471

10. Ogasawara S, Chiba T, Ooka $\mathrm{Y}$, et al. A randomized placebo-controlled trial of prophylactic dexamethasone for transcatheter arterial chemoembolization. Hepatology. 2018;67(2):575-585. doi:10.1002/hep.v67.2

11. Andersen KJ, Gronbaek H, Villadsen GE, et al. Chemoembolization of intermediate stage hepatocellular carcinomas: results from a Nordic tertiary liver cancer center. Indian $J$ Gastroenterol. 2014;33(4):322-329. doi:10.1007/s12664-013-0428-9

12. Guo J, Zhao L, Rao Y, et al. Novel multimodal analgesia regimen improves post-tace pain in patients with hepatocellular carcinoma Hepatobiliary Pancreat Dis Int. 2018;17(6):510-516. doi:10.1016/j. hbpd.2018.08.001

13. Benzakoun J, Ronot M, Lagadec M, et al. Risks factors for severe pain after selective liver transarterial chemoembolization. Liver Int. 2017;37(4):583-591. doi:10.1111/liv.2017.37.issue-4

14. Khalaf MH, Sundaram V, AbdelRazek MM, et al. A predictive model for postembolization syndrome after transarterial hepatic chemoembolization of hepatocellular carcinoma. Radiology. 2019;290 (1):254-261. doi:10.1148/radiol.2018180257
15. Patel NH, Hahn D, Rapp S, Bergan K, Coldwell DM. Hepatic artery embolization: factors predisposing to postembolization pain and nausea. J Vasc Interv Radiol. 2000;11(4):453-460. doi:10.1016/ S1051-0443(07)61377-3

16. Xiao YD, Ma C, Zhang ZS, Liu J. Safety and efficacy assessment of transarterial chemoembolization using drug-eluting beads in patients with hepatocellular carcinoma and arterioportal shunt: a single-center experience. Cancer Manag Res. 2019;11:1551-1557. doi:10.2147/ CMAR.S193948

17. Zhou GH, Han J, Sun JH, et al. Efficacy and safety profile of drugeluting beads transarterial chemoembolization by CalliSpheres ${ }^{\mathbb{R}}$ beads in Chinese hepatocellular carcinoma patients. BMC Cancer. 2018;18(1):644. doi:10.1186/s12885-018-4566-4

18. Song MJ, Chun HJ, Song DS, et al. Comparative study between doxorubicin-eluting beads and conventional transarterial chemoembolization for treatment of hepatocellular carcinoma. $J$ Hepatol. 2012;57(6):1244-1250. doi:10.1016/j.jhep.2012.07.017

19. Miyayama S, Matsui O. Superselective conventional transarterial chemoembolization for hepatocellular carcinoma: rationale, technique, and outcome. J Vasc Interv Radiol. 2016;27(9):1269-1278. doi:10.1016/j.jvir.2016.04.014

20. Wang ZX, Liu SL, Sun CH, Wang Q. Psychological intervention reduces postembolization pain during hepatic arterial chemoembolization therapy: a complementary approach to drug analgesia. World J Gastroenterol. 2008;14(6):931-935. doi:10.3748/wjg.14.931

21. Hawker GA, Mian S, Kendzerska T, French M. Measures of adult pain: visual Analog Scale for Pain (VAS Pain), Numeric Rating Scale for Pain (NRS Pain), McGill Pain Questionnaire (MPQ), Short-Form McGill Pain Questionnaire (SF-MPQ), Chronic Pain Grade Scale (CPGS), Short Form-36 Bodily Pain Scale (SF-36 BPS), and Measure of Intermittent and Constant Osteoarthritis Pain (ICOAP). Arthritis Care Res. 2011;63:S240-S252.

22. Ma JB, Wang FM, Zhang WQ, et al. Percutaneous cryoablation for the treatment of liver cancer at special sites: an assessment of efficacy and safety. Quant Imaging Med Surg. 2019;9:1948-1957. doi:10.21037/qims.2019.11.12

23. Gaba RC, Lewandowski RJ, Hickey R, et al. Transcatheter therapy for hepatic malignancy: standardization of terminology and reporting criteria. J Vasc Interv Radiol. 2016;27(4):457-473. doi:10.1016/j. jvir.2015.12.752

24. Leung DA, Goin JE, Sickles C, Raskay BJ, Soulen MC. Determinants of postembolization syndrome after hepatic chemoembolization. J Vasc Interv Radiol. 2001;12(3):321-326. doi:10.1016/S1051-0443(07)61911-3

25. Hinrichs JB, Hasdemir DB, Nordlohne M, Schweitzer N, Wacker F, Vogel A. Health-related quality of life in patients with hepatocellular carcinoma treated with initial transarterial chemoembolization. Cardiovasc Intervent Radiol. 2017;40(10):1559-1566. doi:10.1007/ s00270-017-1681-6

26. Getzin T, Gueler F, Hartleben B, et al. Gd-EOB-DTPA-enhanced MRI for quantitative assessment of liver organ damage after partial hepatic ischaemia reperfusion injury: correlation with histology and serum biomarkers of liver cell injury. Eur Radiol. 2018;28 (10):4455-4464. doi:10.1007/s00330-018-5380-8

27. Lv WF, Liu KC, Lu D, et al. Transarterial chemoembolization for hepatocellular carcinoma combined with portal vein tumor thrombosis. Cancer Manag Res. 2018;10:4719-4726. doi:10.2147/CMAR.S166527

28. Lima M, Dutra S, Gomes FV, Bilhim T, Coimbra E. Risk factors for the development of postembolization syndrome after transarterial chemoembolization for hepatocellular carcinoma treatment. Acta Med Port. 2018;31(1):22-29. doi:10.20344/amp.8976

29. Mason MC, Massarweh NN, Salami A, Sultenfuss MA, Anaya DA. Post-embolization syndrome as an early predictor of overall survival after transarterial chemoembolization for hepatocellular carcinoma. HPB. 2015;17(12):1137-1144. doi:10.1111/hpb.12487 
Journal of Pain Research

Dovepress

\section{Publish your work in this journal}

The Journal of Pain Research is an international, peer reviewed, open access, online journal that welcomes laboratory and clinical findings in the fields of pain research and the prevention and management of pain. Original research, reviews, symposium reports, hypothesis formation and commentaries are all considered for publication. The manuscript management system is completely online and includes a very quick and fair peer-review system, which is all easy to use. Visit http:// www.dovepress.com/testimonials.php to read real quotes from published authors.

Submit your manuscript here: https://www.dovepress.com/journal-of-pain-research-journal 